\title{
Activation of AMP-Activated Protein Kinase Attenuates Tumor Necrosis Factor- $\alpha$-Induced Lipolysis via Protection of Perilipin in 3T3-L1 Adipocytes
}

\author{
Seok-Woo Hong ${ }^{1}$, Jinmi Lee ${ }^{1}$, Se Eun Park², Eun-Jung Rhee², Cheol-Young Park², Ki-Won Oh², Sung-Woo Park², \\ Won-Young Lee ${ }^{2}$ \\ ${ }^{1}$ Institute of Medical Research, ${ }^{2}$ Division of Endocrinology and Metabolism, Department of Internal Medicine, Kangbuk \\ Samsung Hospital, Sungkyunkwan University School of Medicine, Seoul, Korea
}

Background: Tumor necrosis factor (TNF)- $\alpha$ and AMP-activated protein kinase (AMPK) are known to stimulate and repress lipolysis in adipocytes, respectively; however, the mechanisms regulating these processes have not been completely elucidated.

Methods: The key factors and mechanism of action of TNF- $\alpha$ and AMPK in lipolysis were investigated by evaluating perilipin expression and activity of protein kinase RNA-like endoplasmic reticulum kinase (PERK)/eukaryotic initiation factor $2 \alpha$ (eIF2 $\alpha$ ) by Western blot and an immunofluorescence assay in 24-hour TNF- $\alpha$-treated 3T3-L1 adipocytes with artificial manipulation of AMPK activation.

Results: Enhancement of AMPK activity by the addition of activator minoimidazole carboxamide ribonucleotide (AICAR) suppressed TNF- $\alpha$-induced lipolysis, whereas the addition of compound C, an inhibitor of AMPK phosphorylation, enhanced lipolysis. Perilipin, a lipid droplet-associated protein, was decreased by TNF- $\alpha$ and recovered following treatment with AICAR, showing a correlation with the antilipolytic effect of AICAR. Significant activation of PERK/eIF2 $\alpha$, a component of the unfolded protein response signaling pathway, was observed in TNF- $\alpha$ or vesicle-treated 3T3-L1 adipocytes. The antilipolytic effect and recovery of perilipin expression by AICAR in TNF- $\alpha$-treated 3T3-L1 adipocytes were significantly diminished by treatment with 2-aminopurine, a specific inhibitor of eIF $2 \alpha$.

Conclusion: These data indicated that AICAR-induced AMPK activation attenuates TNF- $\alpha$-induced lipolysis via preservation of perilipin in 3T3-L1 adipocytes. In addition, PERK/ eIF2 $\alpha$ activity is a novel mechanism of the anti-lipolytic effect of AICAR.

Keywords: AMP-activated protein kinases; Lipolysis; Perilipin; PERK/eIF2 $\alpha$; Adipocytes

\section{INTRODUCTION}

Tumor necrosis factor (TNF)- $\alpha$ is a cytokine with a well-established role in immunomodulatory and inflammatory responses, and has been implicated in the development of obesity [1,2]. Elevated TNF- $\alpha$ production in adipose tissues and adipocytes from obese subjects suggests that TNF- $\alpha$ may be linked to diabetes and insulin resistance [3-5]. Several laboratories have demon-
Received: 27 February 2014, Revised: 11 April 2014,

Accepted: 24 April 2014

Corresponding author: Won-Young Lee

Division of Endocrinology and Metabolism, Department of Internal Medicine, Kangbuk Samsung Hospital, Sungkyunkwan University School of Medicine, 29 Saemunan-ro, Jongno-gu, Seoul 110-746, Korea

Tel: +82-2-2001-2579, Fax: +82-2-2001-2049, E-mail: drlwy@hanmail.net

\section{Copyright $\odot 2014$ Korean Endocrine Society}

This is an Open Access article distributed under the terms of the Creative Commons Attribution Non-Commercial License (http://creativecommons.org/ licenses/by-nc/3.0/) which permits unrestricted non-commercial use, distribution, and reproduction in any medium, provided the original work is properly cited. 
strated that TNF- $\alpha$ increases lipolysis and release of free fatty acids (FFAs) from adipocytes [6-8]. In addition, it has been shown that the levels of TNF- $\alpha$ and FFAs are positively correlated in adipose tissues [9]. However, the mechanism of TNF- $\alpha$ function in obese subjects and lipolysis is not completely understood.

AMP-activated protein kinase (AMPK) is a widely-expressed serine/threonine kinase that is considered to act as an intracellular energy sensor. Because AMPK activation may have beneficial metabolic consequences in diabetic patients, AMPK has emerged as a potential target for the treatment of obesity and type 2 diabetes [10]. It has been demonstrated that two classes of antidiabetic drugs (metformin and thiazolidinediones) act at least in part through activation of AMPK in liver and muscle $[11,12]$. Whereas the function of AMPK in liver and muscle has been well-illustrated, the role of AMPK in adipose tissue remains poorly documented. An antilipolytic effect of the AMPK activator minoimidazole carboxamide ribonucleotide (AICAR) has been demonstrated in a lipolysis model treated with cyclic AMP (cAMP) inducers $[13,14]$. However, although the cAMP level is increased in TNF- $\alpha$-treated adipocytes, the main mechanism of TNF- $\alpha$-induced lipolysis has remained unknown.

In adipocytes, induction of stress can increase the expression and secretion of proinflammatory cytokines [15]. Overproduction and chronic exposure to proinflammatory cytokines such as TNF- $\alpha$ and interleukin- 6 can induce lipolysis and apoptosis in adipocytes as a result of endoplasmic reticulum (ER) stress and generation of reactive oxygen species [16]. A variety of environmental insults leads to the phosphorylation of a family of proteins associated with the unfolded protein response (UPR), including protein kinase RNA-like endoplasmic reticulum kinase (PERK) and eukaryotic initiation factor $2 \alpha$ (eIF2 $\alpha$ ), to alleviate cellular injury, or alternatively induce apoptosis. Phosphorylation of eIF $2 \alpha$ regulates global translation, allowing cells to conserve resources and to initiate the reconfiguration of gene expression to effectively manage stress conditions [17].

The aim of this study was to determine the mechanism of TNF- $\alpha$-induced lipolysis and the antilipolytic effect of AMPK in fully differentiated 3T3-L1 adipocytes. We identified the key factor in TNF- $\alpha$-induced lipolysis, and also investigated the effect of TNF- $\alpha$ and AMPK on factors relating to ER stress and the UPR.

\section{METHODS}

\section{Materials}

TNF- $\alpha$ and 2-aminopurine (2-AP) were purchased from Sigma
Chemical (St. Louis, MO, USA). AICAR and compound C (CC) were purchased from Calbiochem (San Diego, CA, USA). Antibodies against AMPK, phosphorylated-AMPK, $\beta$-actin, perilipin, hormone sensitive lipase (HSL), eIF $2 \alpha$, phosphosphorylated-eIF2 $\alpha$, PERK, and phosphorylated-PERK were purchased from Cell Signaling (Beverly, MA, USA).

\section{Cell cultures}

3T3-L1 fibroblasts were cultured in high-glucose Dulbecco's modified Eagle medium (DMEM) supplemented with 10\% fetal calf serum, $50 \mu \mathrm{g} / \mathrm{mL}$ of penicillin, and $50 \mu \mathrm{g} / \mathrm{mL}$ of streptomycin. After the fibroblasts reached quiescence, adipocyte differentiation was induced by adding methylisobutylxanthine (100 $\mu \mathrm{M})$, dexamethasone $(0.25 \mu \mathrm{M})$, and insulin $(1 \mu \mathrm{g} / \mathrm{mL})$ for 2 days. Cells were cultured in high-glucose DMEM supplemented with $10 \%$ calf serum and insulin for an additional 3 days, then maintained in high-glucose DMEM only supplemented with $10 \%$ fetal bovine serum. 3T3-L1 adipocytes were used for experiments 8 to 10 days after differentiation and were treated as described below for mature adipocytes.

\section{Lipolysis assays}

Lipolysis was stimulated by incubation with TNF- $\alpha(10 \mu \mathrm{g} / \mathrm{mL})$ for 24 hours in the presence or absence of AICAR $(1 \mu \mathrm{M})$ or CC $(20 \mu \mathrm{M})$, an AMPK activator and inhibitor, respectively. Lipolysis was assessed from the release of glycerol in the culture medium, as previously described [18] using free glycerol reagent (Sigma, St. Louis, MO, USA). Lipolysis of adipocytes treated with 2-AP ( $5 \mathrm{mM})$, a specific inhibitor of eIF2 $\alpha$ kinase, was also assessed to evaluate the relationship between the PERK/eIF2 $\alpha$ pathway and lipolysis.

\section{Western blot analysis}

Cultured cells were scraped and lysed on ice in radioimmunoprecipitation assay buffer (Santa Cruz Biochemistry, Santa Cruz, CA, USA). After lysis for 30 minutes, protein was extracted by centrifugation at $13,000 \mathrm{rpm}$ for 20 minutes at $4^{\circ} \mathrm{C}$. The samples containing $30 \mu \mathrm{g}$ of protein were separated on $4 \%$ to $12 \%$ bis-Tris Nupage gels (Invitrogen, Carlsbad, CA, USA), then transferred to polyvinylidene difluoride (PVDF) membranes. The PVDF membranes were incubated overnight at $4^{\circ} \mathrm{C}$ with primary antibodies against molecules of interest. After an additional incubation for 1 hour at room temperature, membranes were incubated with horseradish peroxidase-conjugated secondary antibodies, and visualized using enhanced chemiluminescence Western blotting detection reagents. 


\section{Immunofluorescence assay}

For determination of perilipin protein, 3T3-L1 adipocytes were cultured on cover glass-bottomed dishes and incubated with reagents, as described above. After treatment, cells were fixed with $4 \%$ paraformaldehyde for 5 minutes at room temperature, washed, and incubated with polyclonal antibodies against perilipin overnight. For fluorescence detection, fluorescein isothiocyanate-conjugated immunoglobulin $\mathrm{G}$ was used after incubation with primary antibodies. Nuclei were counterstained with DAPI (Invitrogen).

\section{Statistical analysis}

The data are presented as the mean \pm SD. Significance was assessed by Student two sample $t$ tests. A $P$ value of less than 0.05 was considered significant.

\section{RESULTS}

\section{AICAR inhibits TNF- $\alpha$-induced lipolysis in 3T3-L1 adipocytes}

To evaluate the role of AMPK in the regulation of lipolysis, we first confirmed spontaneous phosphorylation of AMPK in adipocytes which were exposed to TNF- $\alpha$ for 24 hours. Phosphorylation of the $\alpha$-subunit of AMPK at the critical activating threonine residue, Thr-172, was evaluated by immunoreactivity with a specific antibody for the phosphorylated AMPK protein (Fig. 1A). The phosphorylation of AMPK was controlled by pre- and co-treatment with chemical reagents, showing a remarkable increase and decrease by AICAR and $\mathrm{CC}$, respectively. In fullydifferentiated 3T3-L1 adipocytes, treatment with TNF- $\alpha$ and manipulation of AMPK activity resulted in an alteration of lipolysis, as measured by the amount of released glycerol. As shown in Fig. 1B, lipolysis was significantly elevated to approximately 1.8 -fold in cells cultured in DMEM containing 10 $\mathrm{ng} / \mathrm{mL}$ of TNF- $\alpha$, and accelerated 2.3 -fold by blocking of AMPK activity. Concordantly, TNF- $\alpha$-induced lipolysis was abrogated upon activation of AMPK by AICAR treatment.

\section{AMPK alleviates TNF- $\alpha$ induced diminution of perilipin}

Perilipin, which protects lipids on the surface of lipid droplets, is a key factor in the process of lipolysis by lipolytic enzymes. The amount of perilipin protein was decreased in TNF- $\alpha$ treated adipocytes, and was further diminished by treatment with $\mathrm{CC}$, while perilipin protein was restored in adipocytes incubated with TNF- $\alpha$ and AICAR (Fig. 2A, B). In contrast, the amount of HSL was unchanged in TNF- $\alpha$ and/or AMPK-regu-
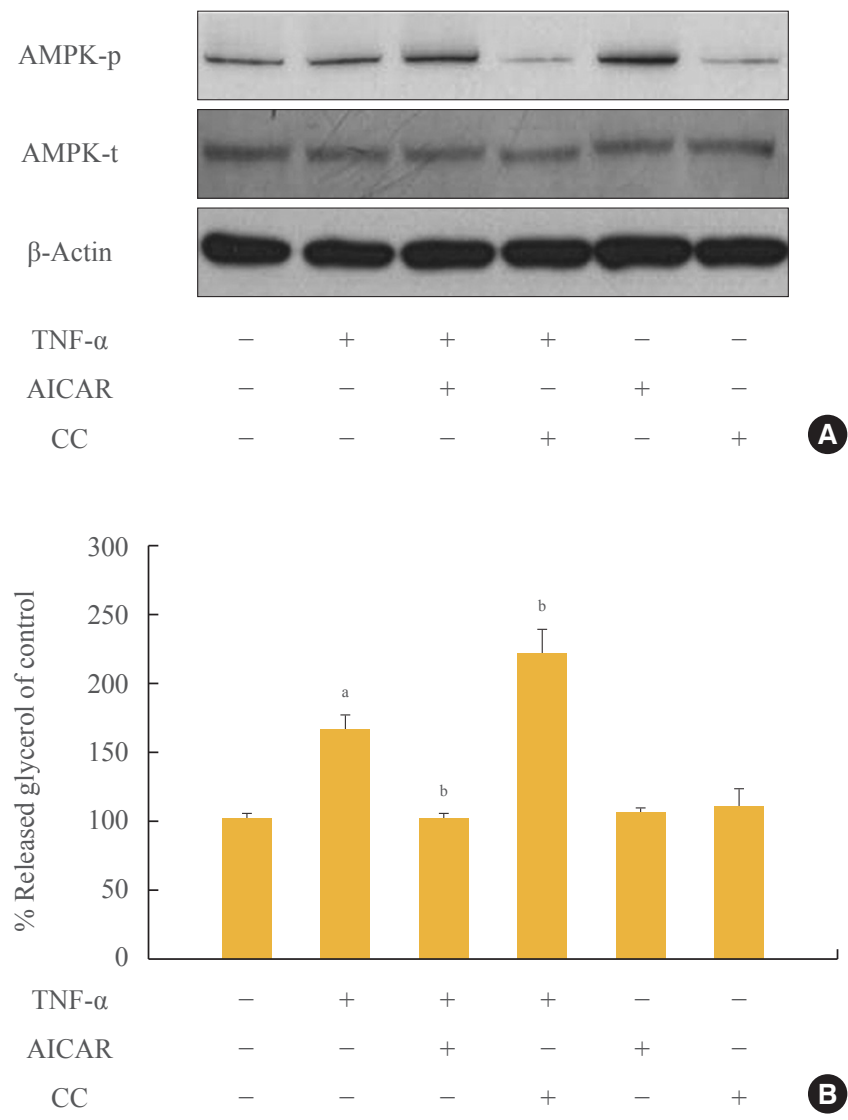

Fig. 1. Chronic incubation with tumor necrosis factor (TNF)- $\alpha$ and AMP-activated protein kinase (AMPK) activation regulates lipolysis in cultured 3T3-L1 adipocytes. Adipocytes were incubated with or without TNF- $\alpha(10 \mathrm{ng} / \mathrm{mL})$ for 24 hours in the presence or absence of activator minoimidazole carboxamide ribonucleotide (AICAR; $1 \mathrm{mM}$ ) or compound C (CC; $20 \mu \mathrm{M})$. (A) Total and phosphorylated AMPK protein levels were examined by Western blot assay. (B) Lipolysis was quantified by determination of glycerol release into the media. Aliquots of the culture medium were collected at 24 hours, and the amount of released glycerol was measured. The results represent the mean $\pm \mathrm{SE}$ from at least three independent batches of 3T3-L1 adipocytes. The released glycerol level in control adipocytes was designated as $100 \%$. ${ }^{\mathrm{a}} P<0.001$ compared with the control group; ${ }^{\mathrm{b}} P<0.01$ compared with the TNF- $\alpha$ group.

lated adipocytes. We also evaluated the amount of perilipin protein using an immunofluorescence assay (Fig. 2C). The intensity of green fluorescence indicating perilipin protein on the surface of lipid droplets decreased upon TNF- $\alpha$ and/or CC treatment. In adipocytes incubated with AICAR and TNF- $\alpha$; however, green fluorescence was remarkably increased in amount and intensity, although it was weaker than the level of intensity in control adipocytes. 

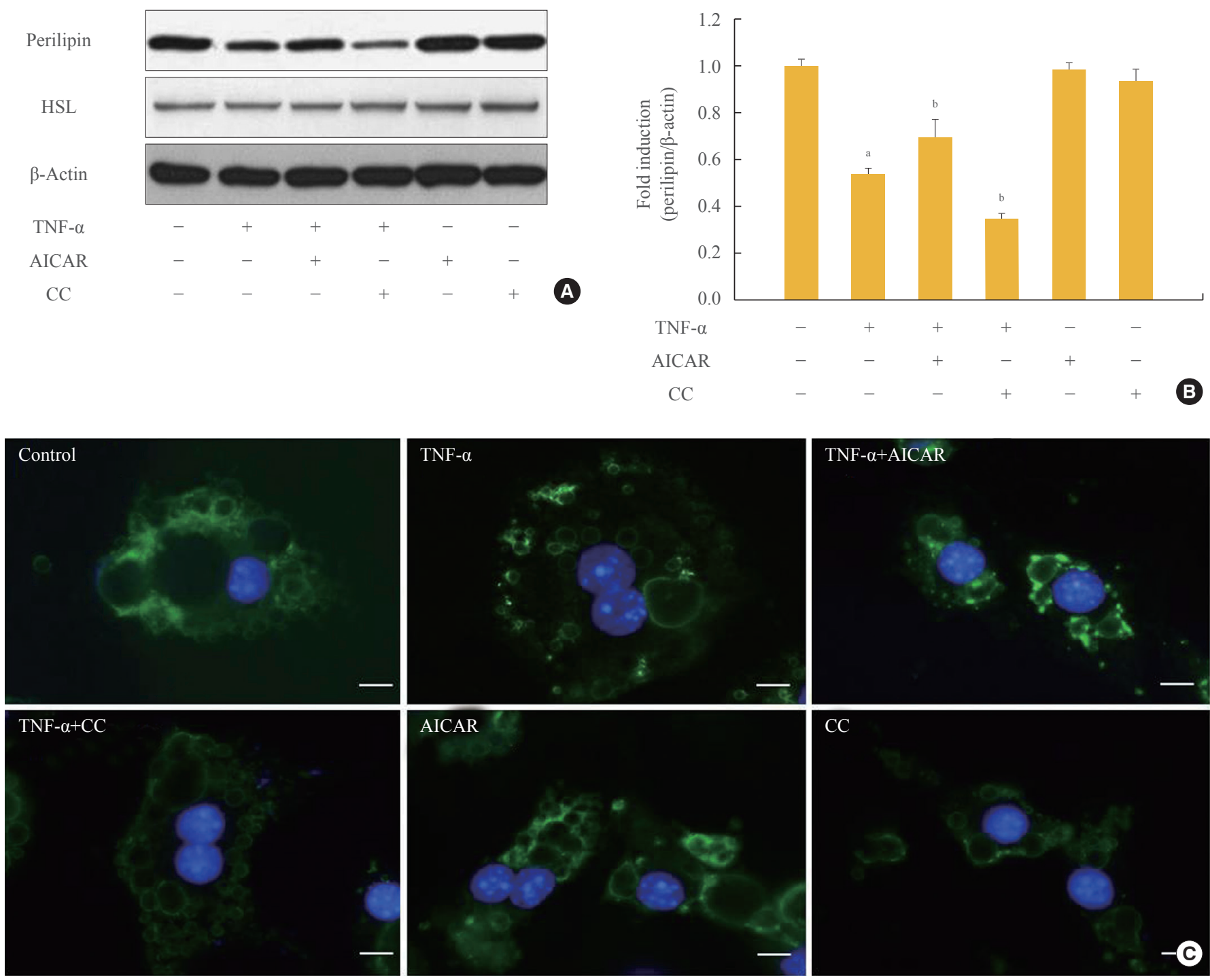

Fig. 2. AMP-activated protein kinase (AMPK) attenuates the tumor necrosis factor (TNF)- $\alpha$-induced decrease in perilipin. 3T3-L1 adipocytes were incubated with TNF- $\alpha(10 \mathrm{ng} / \mathrm{mL})$ for 24 hours in the presence or absence of activator minoimidazole carboxamide ribonucleotide (AICAR; $1 \mathrm{mM}$ ) or compound C (CC; $20 \mu \mathrm{M})$. (A) Perilipin and hormone sensitive lipase (HSL) protein levels were examined by Western blot assay. (B) Quantification of blot shown in (A). (C) At the end of the incubation, cells were fixed, permeabilized, and then incubated with a specific antibody against perilipin (green fluorescence). Nuclei were visualized by DAPI staining (blue fluorescence). Bars $=10 \mu \mathrm{m}$. ${ }^{\mathrm{a}} P<0.001$ compared with control group; ${ }^{\mathrm{b}} P<0.01$ compared with TNF- $\alpha$ group.

\section{AMPK stimulates phosphorylation of PERK/eIF2 $\alpha$ in TNF- $\alpha$-treated adipocytes}

There is increasing evidence showing that UPR signaling, including PERK and eIF2 $\alpha$, is linked to adipocyte dysfunction $[16,19,20]$. Although PERK/eIF2 $\alpha$ was not affected by TNF- $\alpha$ treatment in 3T3-L1 adipocytes, stimulation of AMPK with AICAR treatment significantly increased the activation of PERK/eIF2 $\alpha$ in 3T3-L1 adipocytes, with or without TNF- $\alpha$ treatment (Fig. 3).

\section{Antilipolytic effect of AMPK is dependent on UPR}

Next we investigated the involvement of the PERK/eIF2 $\alpha$ pathway on the antilipolytic effect of AICAR, as described by perilipin protein and the amount of released glycerol. To this end, we used a concentration of $5 \mathrm{mM} 2-\mathrm{AP}$, which inhibits eIF2 $\alpha$ kinase, to repress AICAR-induced PERK/eIF2 $\alpha$ activation. Restoration of perilipin protein by AICAR was completely abolished by 2-AP (Fig. 4A, B). Supplementation of adipocytes with 2-AP significantly overcame the antilipolytic effect of AICAR, describing a significant increase in lipolysis. 

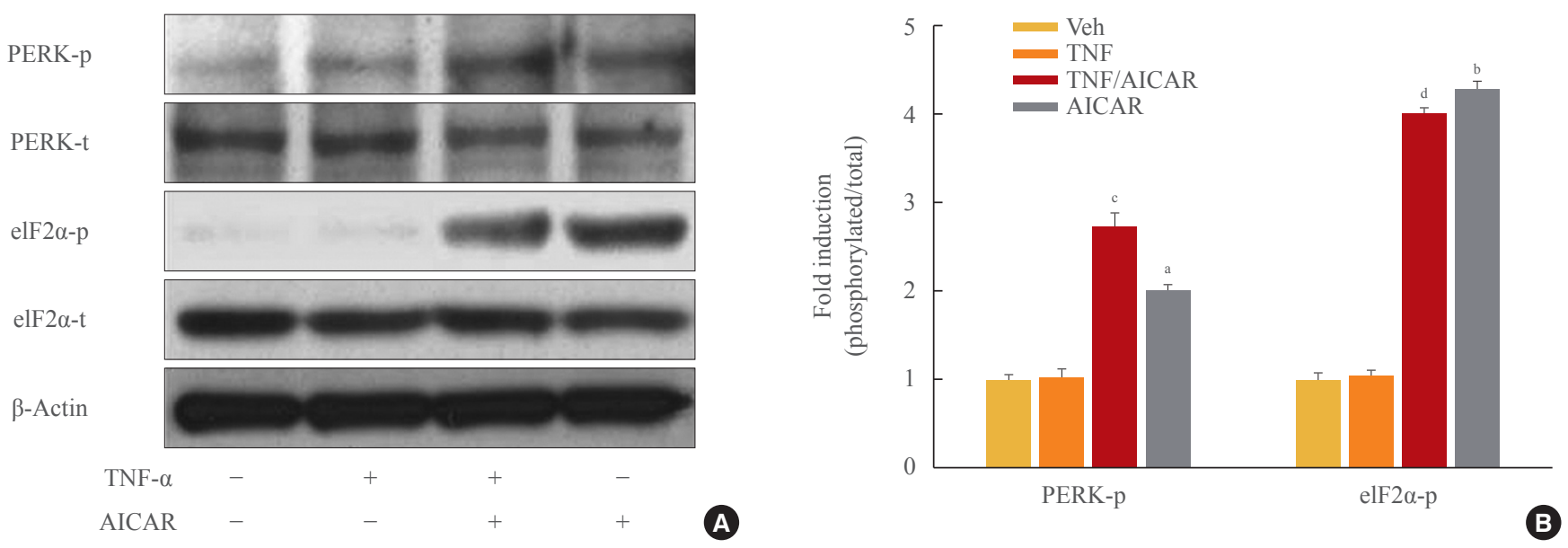

Fig. 3. AMP-activated protein kinase (AMPK) stimulates phosphorylation of protein kinase RNA-like endoplasmic reticulum kinase (PERK)/eukaryotic initiation factor $2 \alpha$ (eIF2 $\alpha)$. 3T3-L1 adipocytes were incubated with tumor necrosis factor (TNF)- $\alpha$ (10 ng/mL) for 24 hours in the presence or absence of activator minoimidazole carboxamide ribonucleotide (AICAR; $1 \mathrm{mM}$ ). (A) Total and phosphorylated form of eIF2 $\alpha$, and PERK protein were detected by Western blot assay. (B) Quantification of blot shown in (A). ${ }^{\mathrm{a}} P<0.01$; and ${ }^{\mathrm{b}} P<0.001$ compared with control group; ${ }^{\mathrm{c}} P<0.01$; and ${ }^{\mathrm{d}} P<0.001$ compared with TNF- $\alpha$ group.

\section{DISCUSSION}

AMPK regulates cellular metabolism, as well as responses to a variety of signals not directly related to metabolism, such as ischemia [21], hypoxia [22,23], and oxidative stress [24]. In adipocytes it has been reported that AMPK is involved in triglyceride breakdown [25], with most reports indicating that AMPK antagonizes cAMP-mediated lipolysis [14,26]. Recently, it has also been reported that a key factor in the antilipolytic effect of AMPK is HSL in cAMP-dependent lipolysis in mature adipocytes [18]. However, because TNF- $\alpha$-induced lipolysis is mediated by an intracellular pathway distinct from cAMP-dependent lipolysis, it is difficult to fully explain the mechanism of antilipolysis.

Here we demonstrated an increase in phosphorylation of AMPK by TNF- $\alpha$ in adipocytes. We also showed that additional enhancement of AMPK activity by artificial manipulation repressed TNF- $\alpha$-induced lipolysis (Fig. 1). These findings imply that an increase in AMPK activity in TNF- $\alpha$-treated adipocytes is an antagonistic response to resist the lipolytic effect of TNF- $\alpha$. TNF- $\alpha$ can stimulate lipolysis by at least three separate mechanisms in adipocytes: inhibiting insulin receptor signaling, thereby counteracting the antilipolytic effect of the hormone; inhibiting signaling through the Gi-protein-coupled adenosine receptor to counteract the antilipolytic effect of adenosine; and stimulation of basal (nonhormonal) lipolysis through a decrease in the lipid-binding protein, perilipin [27]. In the present study, we found quantitative and morphologic alteration of perilipin by TNF- $\alpha$ and AMPK. In addition, lipolysis was directly affected by fluctuation of perilipin expression (Fig. 2). We concluded that perilipin is an essential factor in TNF- $\alpha$-induced lipolysis, and that AMPK represses lipolysis via preservation of perilipin.

In response to a variety of environmental insults such as accumulation of misfolded proteins, a family of protein kinases, including PERK and eIF2 $\alpha$, is phosphorylated to alleviate cellular injury or induce apoptosis. Phosphorylation of PERK/ eIF2 $\alpha$ is a pivotal process of the UPR and reduces global translation, allowing cells to conserve resources and initiate the reconfiguration of gene expression to effectively manage stress conditions [17]. We therefore investigated variation in perilipin expression under conditions that inhibit eIF2 $\alpha$ phosphorylation to determine whether PERK/eIF2 $\alpha$ signaling induced lipolysis in adipocytes. We used 2-AP, a specific inhibitor of PERK/ eIF2 $\alpha$ phosphorylation, to damage the UPR mechanism, which manages cell stress. In adipocytes treated with TNF- $\alpha$, activation of AMPK by AICAR preserved perilipin expression with stimulation of eIF $2 \alpha$ phosphorylation. However, AICAR-induced preservation of perilipin was greatly diminished in 2-AP-treated adipocytes, which showed an increase in lipolysis (Fig. 4). Blockade of PERK/eIF2 $\alpha$ signaling completely abrogated protection of perilipin and repression of lipolysis by AICAR. These data suggested that PERK/eIF2 $\alpha$ plays a central role in the antilipolytic effect of AMPK in TNF- $\alpha$-induced lipolysis.

AMPK has been investigated as an essential target molecule 

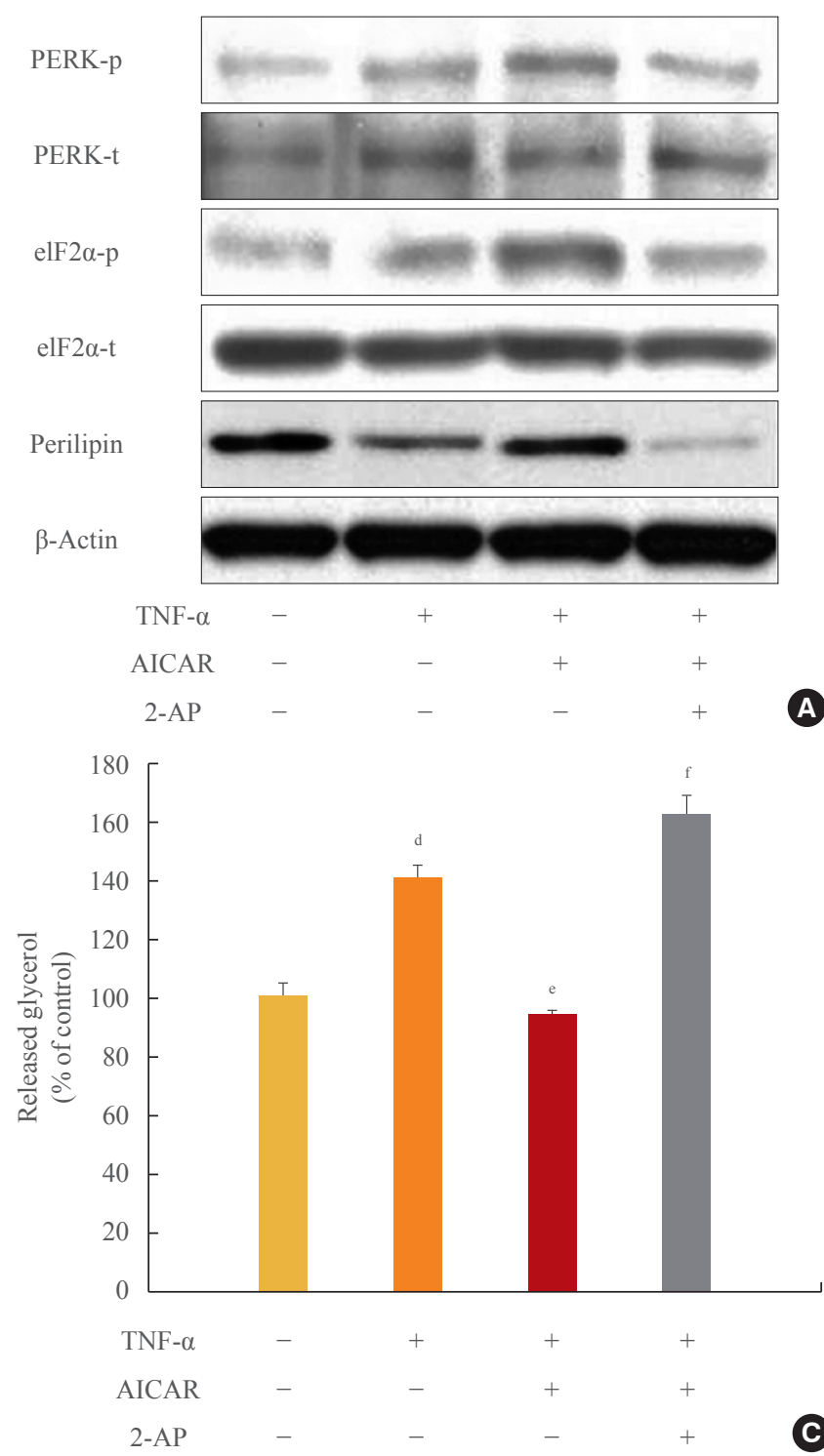

in a number of organs and cells, including pancreatic $\beta$-cells, muscle, liver, and brain, to cure diabetes and obesity [28]. TNF- $\alpha$ is one of the etiologic factors in a variety of diseases, and it is increased in obese individuals. In this study, we demonstrated an antilipolytic effect of AMPK in TNF- $\alpha$-induced adipocytes through regulation of PERK/eIF2 signaling. The cytoprotective effect of AMPK against TNF- $\alpha$ could be of practical use to treat diabetes and obesity, because the effect is expected to also exist in other organs and cells.

Based on these results, we suggest that AMPK represses TNF- $\alpha$-induced lipolysis via protection of perilipin. It remains to be seen whether pharmacological manipulation of these pathway has the same effect in the therapeutic management of lipolysis in vivo.

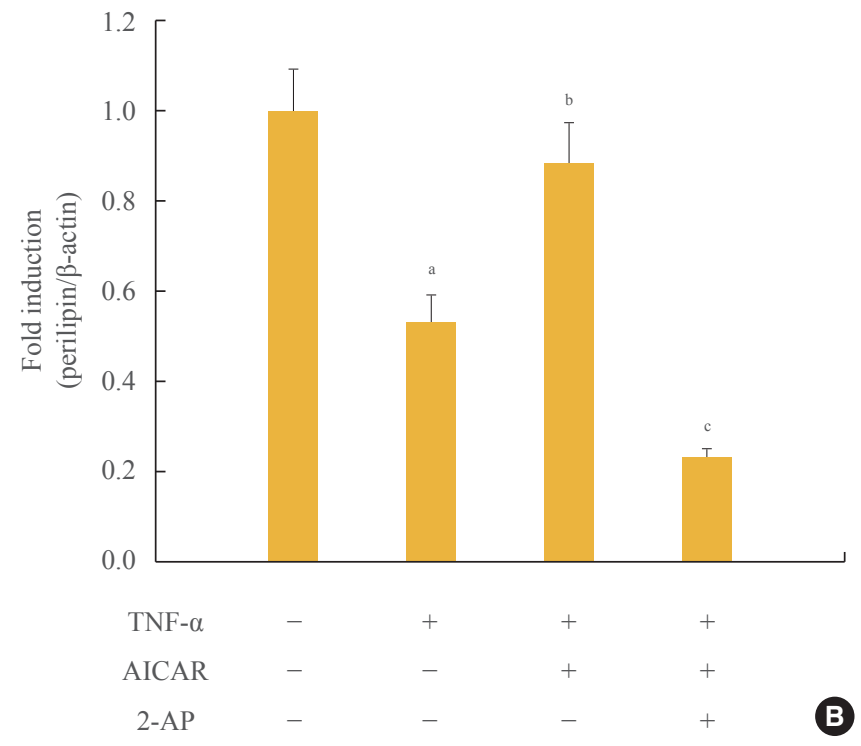

Fig. 4. Antilipolytic effect of AMP-activated protein kinase (AMPK) is abrogated by protein kinase RNA-like endoplasmic reticulum kinase (PERK)/eukaryotic initiation factor $2 \alpha$ (eIF2 $\alpha$ ) inhibition. 3T3-L1 adipocytes were incubated with tumor necrosis factor (TNF)- $\alpha(10 \mathrm{ng} / \mathrm{mL})$ for 24 hours in the presence or absence of activator minoimidazole carboxamide ribonucleotide (AICAR; $1 \mathrm{mM}$ ) and/or 2-aminopurine (2-AP; $5 \mathrm{mM}$ ). (A) Total and phosphorylated form of PERK/eIF $2 \alpha$ and perilipin protein were detected by Western blot assay. (B) Quantification of perilipin protein from the blot shown in (A). (C) At the end of the incubation, the amount of released glycerol in the culture medium was measured. The released glycerol level in control adipocytes was set at $100 \%$. ${ }^{\text {a }} P<0.05$ compared with control group; ${ }^{\mathrm{b}} P<0.05$ compared with TNF- $\alpha$ group; ${ }^{c} P<0.01$ compared with TNF- $\alpha$ and AICAR group; ${ }^{\mathrm{d}} P<0.01$ compared with the control group; ${ }^{\mathrm{e}} P<0.001$ compared with the TNF- $\alpha$ group; ${ }^{\mathrm{f}} P<0.001$ compared with TNF- $\alpha$ and AICAR groups.

\section{CONFLICTS OF INTEREST}

This work was supported by a grant from Chon Kun Dang Pharm (S-2013-0227-000). However the design and interpretation of the work are independent of the funding.

\section{ACKNOWLEDGMENTS}

This work was supported by a National Research Foundation of Korea (NRF) grant funded by the Korea government (NRF2013R1A1A2063069). 


\section{REFERENCES}

1. Qi C, Pekala PH. Tumor necrosis factor-alpha-induced insulin resistance in adipocytes. Proc Soc Exp Biol Med 2000; 223:128-35.

2. Hausman DB, DiGirolamo M, Bartness TJ, Hausman GJ, Martin RJ. The biology of white adipocyte proliferation. Obes Rev 2001;2:239-54.

3. Hotamisligil GS, Shargill NS, Spiegelman BM. Adipose expression of tumor necrosis factor-alpha: direct role in obesity-linked insulin resistance. Science 1993;259:87-91.

4. Hotamisligil GS, Arner P, Caro JF, Atkinson RL, Spiegelman BM. Increased adipose tissue expression of tumor necrosis factor-alpha in human obesity and insulin resistance. J Clin Invest 1995;95:2409-15.

5. Kern PA, Saghizadeh M, Ong JM, Bosch RJ, Deem R, Simsolo RB. The expression of tumor necrosis factor in human adipose tissue: regulation by obesity, weight loss, and relationship to lipoprotein lipase. J Clin Invest 1995;95: 2111-9.

6. Kawakami M, Murase T, Ogawa H, Ishibashi S, Mori N, Takaku F, Shibata S. Human recombinant TNF suppresses lipoprotein lipase activity and stimulates lipolysis in 3T3L1 cells. J Biochem 1987;101:331-8.

7. Feingold KR, Doerrler W, Dinarello CA, Fiers W, Grunfeld C. Stimulation of lipolysis in cultured fat cells by tumor necrosis factor, interleukin-1, and the interferons is blocked by inhibition of prostaglandin synthesis. Endocrinology 1992;130:10-6.

8. Hauner H, Petruschke T, Russ M, Rohrig K, Eckel J. Effects of tumour necrosis factor alpha (TNF alpha) on glucose transport and lipid metabolism of newly-differentiated human fat cells in cell culture. Diabetologia 1995;38:764-71.

9. Orban Z, Remaley AT, Sampson M, Trajanoski Z, Chrousos GP. The differential effect of food intake and beta-adrenergic stimulation on adipose-derived hormones and cytokines in man. J Clin Endocrinol Metab 1999;84:2126-33.

10. Ruderman N, Prentki M. AMP kinase and malonyl-CoA: targets for therapy of the metabolic syndrome. Nat Rev Drug Discov 2004;3:340-51.

11. Zhou G, Myers R, Li Y, Chen Y, Shen X, Fenyk-Melody J, Wu M, Ventre J, Doebber T, Fujii N, Musi N, Hirshman MF, Goodyear LJ, Moller DE. Role of AMP-activated protein kinase in mechanism of metformin action. J Clin Invest 2001;108:1167-74.

12. Fryer LG, Parbu-Patel A, Carling D. The Anti-diabetic drugs rosiglitazone and metformin stimulate AMP-activat- ed protein kinase through distinct signaling pathways. J Biol Chem 2002;277:25226-32.

13. Yin W, Mu J, Birnbaum MJ. Role of AMP-activated protein kinase in cyclic AMP-dependent lipolysis In 3T3-L1 adipocytes. J Biol Chem 2003;278:43074-80.

14. Daval M, Diot-Dupuy F, Bazin R, Hainault I, Viollet B, Vaulont S, Hajduch E, Ferre P, Foufelle F. Anti-lipolytic action of AMP-activated protein kinase in rodent adipocytes. J Biol Chem 2005;280:25250-7.

15. Xu L, Spinas GA, Niessen M. ER stress in adipocytes inhibits insulin signaling, represses lipolysis, and alters the secretion of adipokines without inhibiting glucose transport. Horm Metab Res 2010;42:643-51.

16. Zhou QG, Zhou M, Lou AJ, Xie D, Hou FF. Advanced oxidation protein products induce inflammatory response and insulin resistance in cultured adipocytes via induction of endoplasmic reticulum stress. Cell Physiol Biochem 2010; 26:775-86.

17. Habinowski SA, Witters LA. The effects of AICAR on adipocyte differentiation of 3T3-L1 cells. Biochem Biophys Res Commun 2001;286:852-6.

18. Koh HJ, Hirshman MF, He H, Li Y, Manabe Y, Balschi JA, Goodyear LJ. Adrenaline is a critical mediator of acute exercise-induced AMP-activated protein kinase activation in adipocytes. Biochem J 2007;403:473-81.

19. Lefterova MI, Mullican SE, Tomaru T, Qatanani M, Schupp M, Lazar MA. Endoplasmic reticulum stress regulates adipocyte resistin expression. Diabetes 2009;58:1879-86.

20. van der Kallen CJ, van Greevenbroek MM, Stehouwer CD, Schalkwijk CG. Endoplasmic reticulum stress-induced apoptosis in the development of diabetes: is there a role for adipose tissue and liver? Apoptosis 2009;14:1424-34.

21. Altarejos JY, Taniguchi M, Clanachan AS, Lopaschuk GD. Myocardial ischemia differentially regulates LKB1 and an alternate 5'-AMP-activated protein kinase kinase. J Biol Chem 2005;280:183-90.

22. Esumi H, Izuishi K, Kato K, Hashimoto K, Kurashima Y, Kishimoto A, Ogura T, Ozawa T. Hypoxia and nitric oxide treatment confer tolerance to glucose starvation in a 5'AMP- activated protein kinase-dependent manner. J Biol Chem 2002;277:32791-8.

23. Marsin AS, Bouzin C, Bertrand L, Hue L. The stimulation of glycolysis by hypoxia in activated monocytes is mediated by AMP-activated protein kinase and inducible 6-phosphofructo-2-kinase. J Biol Chem 2002;277:30778-83.

24. Horie T, Ono K, Nagao K, Nishi H, Kinoshita M, Kawamu- 
ra T, Wada H, Shimatsu A, Kita T, Hasegawa K. Oxidative stress induces GLUT4 translocation by activation of PI3$\mathrm{K} /$ Akt and dual AMPK kinase in cardiac myocytes. J Cell Physiol 2008;215:733-42.

25. Kim SJ, Nian C, McIntosh CH. Activation of lipoprotein lipase by glucose-dependent insulinotropic polypeptide in adipocytes. A role for a protein kinase B, LKB1, and AMPactivated protein kinase cascade. J Biol Chem 2007;282: 8557-67.

26. Dagon Y, Avraham Y, Berry EM. AMPK activation regu- lates apoptosis, adipogenesis, and lipolysis by eIF2alpha in adipocytes. Biochem Biophys Res Commun 2006;340:43-7. 27. Langin D, Arner P. Importance of TNFalpha and neutral lipases in human adipose tissue lipolysis. Trends Endocrinol Metab 2006;17:314-20.

28. Viollet B, Mounier R, Leclerc J, Yazigi A, Foretz M, Andreelli F. Targeting AMP-activated protein kinase as a novel therapeutic approach for the treatment of metabolic disorders. Diabetes Metab 2007;33:395-402. 